\title{
Hyperfine and crystal field interactions in multiferroic $\mathrm{HoCrO}_{3}$
}

\author{
C M N Kumar ${ }^{1,2,3, \dagger}$, Y Xiao ${ }^{1, \ddagger}$, H S Nair ${ }^{4}$, J Voigt $^{1}$, B Schmitz ${ }^{1}$, \\ T Chatterji ${ }^{5}$, N H Jalarvo ${ }^{2,3}$, Th Brückel $^{1}$ \\ ${ }^{1}$ Jülich Centre for Neutron Science JCNS and Peter Grünberg Institut PGI, \\ JARA-FIT, Forschungszentrum Jülich, 52425 Jülich, Germany \\ 2 Jülich Centre for Neutron Science JCNS, Forschungszentrum Jülich GmbH, \\ Outstation at SNS, Oak Ridge National Laboratory, Oak Ridge, Tennessee 37831, \\ United States \\ ${ }^{3}$ Chemical and Engineering Materials Division, Spallation Neutron Source, Oak \\ Ridge National Laboratory, Oak Ridge, Tennessee 37831, United States \\ ${ }^{4}$ Department of Physics, Colorado State University, Fort Collins, CO 80523, USA \\ ${ }^{5}$ Institut Laue-Langevin, BP 156, F-38042 Grenoble Cedex 9, France \\ E-mail: ${ }^{\dagger}$ n.kumar@fz-juelich.de; naveenkumarcm@gmail.com, \\ $\ddagger y \cdot x i a o @ f z-j u e l i c h . d e$
}

\begin{abstract}
We report a comprehensive specific heat and inelastic neutron scattering study to explore the possible origin of multiferroicity in $\mathrm{HoCrO}_{3}$. We have performed specific heat measurements in the temperature range $100 \mathrm{mK}-290 \mathrm{~K}$ and inelastic neutron scattering measurements were performed in the temperature range 1.5$200 \mathrm{~K}$. From the specific heat data we determined hyperfine splitting at $22.5(2) \mu \mathrm{eV}$ and crystal field transitions at 1.379(5) meV, 10.37(4) meV, 15.49(9) meV and 23.44(9) meV, indicating the existence of strong hyperfine and crystal field interactions in $\mathrm{HoCrO}_{3}$. Further, an effective hyperfine field is determined to be 600(3) T. The quasielastic scattering observed in the inelastic scattering data and a large linear term $\gamma=6.3(8) \mathrm{mJmol}^{-1} \mathrm{~K}^{-2}$ in the specific heat is attributed to the presence of short range exchange interactions, which is understood to be contributing to the observed ferroelectricity. Further the nuclear and magnetic entropies were computed to be, $\sim 17.2 \mathrm{Jmol}^{-1} \mathrm{~K}^{-1}$ and $\sim 34 \mathrm{Jmol}^{-1} \mathrm{~K}^{-1}$, respectively. The entropy values are in excellent agreement with the limiting theoretical values. An anomaly is observed in peak position of the temperature dependent crystal field spectra around $60 \mathrm{~K}$, at the same temperature an anomaly in the pyroelectric current is reported. From this we could elucidate a direct correlation between the crystal electric field excitations of $\mathrm{Ho}^{3+}$ and ferroelectricity in $\mathrm{HoCrO}_{3}$. Our present study along with recent reports confirm that $\mathrm{HoCrO}_{3}$, and $R \mathrm{CrO}_{3}$ ( $R=$ Rare earth) in general, possess more than one driving force for the ferroelectricity and multiferroicity.
\end{abstract}

PACS numbers: 75.40.-s, 31.30.Gs, 71.70.Ch, 28.20.Cz 


\section{Introduction}

Perovskite chromites $R \mathrm{CrO}_{3}$, where $R$ is a rare earth element or yttrium are revisited in the recent years as possible multiferroic materials in which multiple ferroic orders such as ferroelectricity and antiferromagnetism coexist as discussed below [1 8 ]. Compared to perovskite manganites, which are well studied in light of multiferroicity, the microscopic physical properties of chromites are not explored in detail and the mechanism for multiferroicity in $\mathrm{HoCrO}_{3}$ is still under debate. The coexistence of ferroelectric and magnetic orders in rare-earth orthochromites was first suggested by Subba Rao et al. [9]. Based on dielectric studies it is reported that the heavy rare earth chromites, $R \mathrm{CrO}_{3}$ $(R=\mathrm{Ho}, \mathrm{Er}, \mathrm{Yb}, \mathrm{Lu})$ undergo a ferroelectric transition in the temperature range $439-485 \mathrm{~K}$ [2]. In a recent article, electrical polarization and magnetodielectric effect studies are reported for polycrystalline $\mathrm{LuCrO}_{3}$ and $\mathrm{ErCrO}_{3}\left[\right.$ [6]. Although both $\mathrm{LuCrO}_{3}$ and $\mathrm{ErCrO}_{3}$ showed the presence of a polar state induced by magnetic ordering below $T_{\mathrm{N}}$, polarization was not affected by applied magnetic fields, so that the magnetoelectric coupling was not evident in these compounds. Further, the magnetodielectric effect observed in the case of $\mathrm{ErCrO}_{3}$ is one order of magnitude higher compared with $\mathrm{LuCrO}_{3}$ reflecting the role of different magnetism of rare-earth cations in ferroelectricity [6].

Recently, Ghosh et al., have studied the ferroelectric properties of polycrystalline $\mathrm{HoCrO}_{3}$ by measuring the thermal variation of pyroelectric current [8]. It was found that pyroelectric current exhibits its maximum value around the antiferromagnetic transition temperature $T_{\mathrm{N}}=140 \mathrm{~K}$, nevertheless, the ferroelectric order temperature which associated with the emergence of spontaneous electric polarization is observed at a higher temperature of $T \approx 240 \mathrm{~K}$. The atypical multiferroic behavior observed in $\mathrm{HoCrO}_{3}$ is argued to be a result of Ho displacements and oxygen octahedral rotations in the non-centrosymmetric $P n a 2_{1}$ space group [4,8, 10, 11]. The role of the rare earth ion in determining the physical properties of chromites $R \mathrm{CrO}_{3}$ was revealed in a recent communication where the origin of ferroelectricity in orthochromites has been attributed to the instability of the symmetric position of the rare earth ion [5]. The interaction between magnetic rare earth and week ferromagnetic $\mathrm{Cr}^{3+}$ ions is the driving force for the breaking of symmetry, and thus the emergence of multiferroic behavior in these systems [5, 7]. Despite a debatable multiferriocity, orthochromites possess a plethora of physical phenomena, providing excellent opportunities to study and understand the basic interactions in materials. The detailed knowledge on the properties of rare earth ion is of particular importance to understand the multiferroicity in rare earth chromites $R \mathrm{CrO}_{3}$.

The rare earth orthochromites crystallize in a distorted orthorhombic perovskite structure with four formula units per unit cell [12 14]. In $\mathrm{HoCrO}_{3}$ the exchange coupling between the $\mathrm{Cr}^{3+}$ nearest neighbors is predominantly antiferromagnetic and they order magnetically below the Néel temperature of $T_{\mathrm{N}}=140 \mathrm{~K}[15]$. On the other hand, earlier reports differ on the aspect of Ho-ordering. Cooperatively induced ordering of Ho in $\mathrm{HoCrO}_{3}$ was reported at $12 \mathrm{~K}$ [15, 16] whereas no ordering was observed by Hornreich 
et.al., down to $1.5 \mathrm{~K}$ [17]. Ferroelectricity is observed in $R \mathrm{CrO}_{3}$ systems only when the $R$ ion is magnetic. This directly suggests the exchange interaction between $\mathrm{Cr}^{3+}$ and $R$ is very important in inducing polarization and warrants the study of the local distortions around the $R$ ion as well as its magnetic properties. Hence, we have chosen $\mathrm{HoCrO}_{3}$ as our subject to investigate the role of rare earth in the magnetic and thermodynamic properties of chromites.

\section{Experimental details}

Polycrystalline $\mathrm{HoCrO}_{3}$ was synthesized by solid state reaction of $\mathrm{Ho}_{2} \mathrm{O}_{3}(3 \mathrm{~N})$ and $\mathrm{Cr}_{2} \mathrm{O}_{3}$ $(4 \mathrm{~N})$ in stoichiometric ratio. The precursors were mixed intimately and subsequently heat treated at $1100{ }^{\circ} \mathrm{C}$ for $48 \mathrm{~h}$. Then, the material was reground and annealed again at $1200{ }^{\circ} \mathrm{C}$ for $24 \mathrm{~h}$. The phase purity of the synthesized powder sample was confirmed by powder x-ray diffraction (PXRD) with $\mathrm{Cu}-K_{\alpha 1}(\lambda=1.54059 \AA)$ radiation, using a Huber x-ray diffractometer (Huber G670) in transmission Guinier geometry. The profiles of the PXRD data were analyzed using the Rietveld method [18] implemented in the FullProf software suit [19]. This confirmed the formation of orthorhombic single phase. The powder was then pressed into pellets and sintered at $1000{ }^{\circ} \mathrm{C}$ for $10 \mathrm{~h}$ for further magnetic and thermal characterization. The heat capacity was measured in the temperature range from $100 \mathrm{mK}$ to $290 \mathrm{~K}$ using a commercial Quantum Design Physical Property Measurement System equipped with a dilution insert. The heat capacity values were extracted using the relaxation method [20]. The background heat capacity of the microcalorimeter and the Apiezon $N$ grease used for thermal conduction was measured before the sample measurement and subtracted from the raw data to obtain the absolute heat capacity of the sample.

Inelastic neutron scattering (INS) measurements were carried out on the BASIS backscattering spectrometer of the Spallation Neutron Source (SNS), Oak Ridge National Laboratory, USA [21]. More details of this measurement is presented in reference [22]. Inelastic neutron scattering experiments were also carried out on the high-resolution time-of-flight spectrometer FOCUS at the Spallation Neutron Source SINQ at PSI, Villigen in Switzerland. The polycrystalline sample was enclosed in an aluminum cylinder (12 $\mathrm{mm}$ diameter, $\sim 45 \mathrm{~mm}$ height) and placed into a He - cryostat and the spectrum was collected using an incident energy of $19.61 \mathrm{meV}$. Additional experiments were performed for the empty container as well as for vanadium to allow the correction of the raw data with respect to background, detector efficiency, absorption and detailed balance according to standard procedures. Inelastic data reduction and analysis was carried out using the software DAVE [23]. 


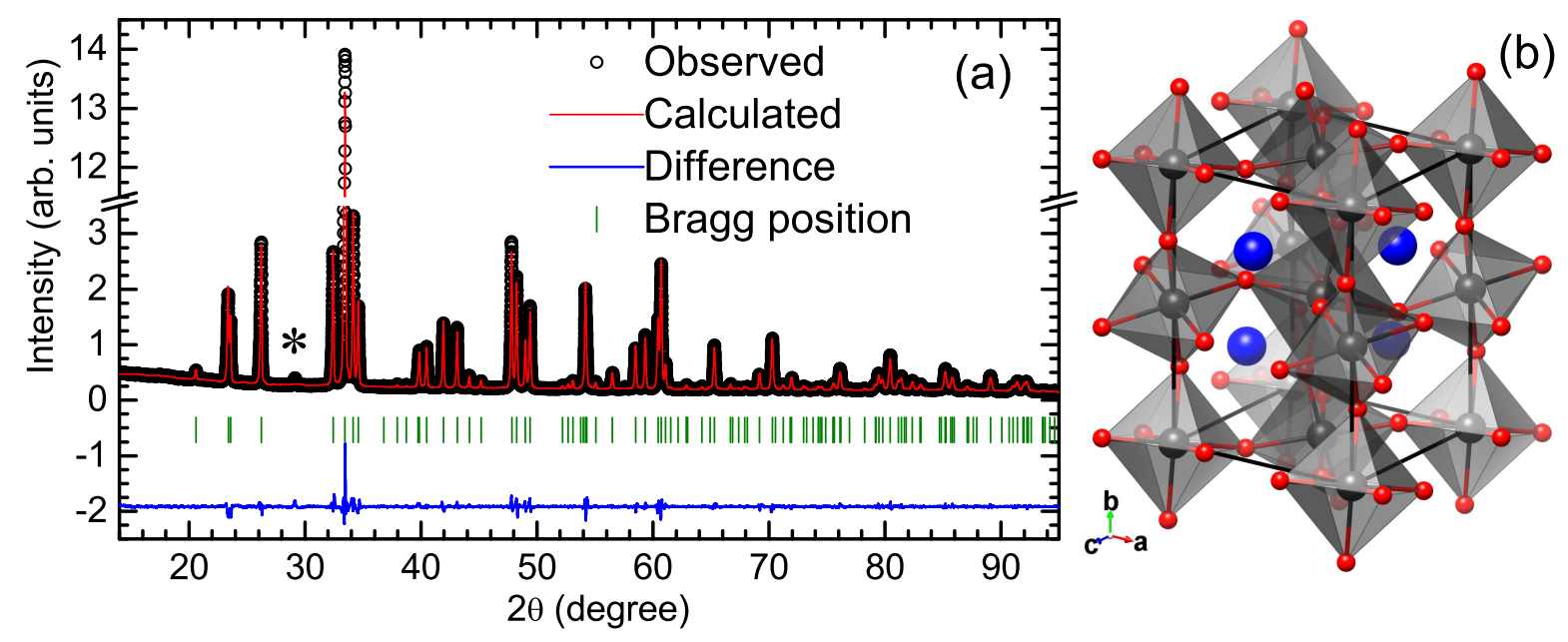

Figure 1: (Color online) Observed (black circle) and calculated (red curve) PXRD patterns and their difference (blue curve) at $300 \mathrm{~K}$. The vertical bars denote the position of Bragg reflections. A spurious peak indicated by asterisk symbol could not be indexed. (b) Graphical representation of the crystal structure of $\mathrm{HoCrO}_{3}$ with $\mathrm{Cr}-\mathrm{O}_{6}$ octahedra in Pbnm space group. Blue, gray and red spheres indicate $\mathrm{Ho}, \mathrm{Cr}$ and $\mathrm{O}$ atoms, respectively.

\section{Results and discussion}

\subsection{Crystal Structure}

The Rietveld refinement of room temperature PXRD data is presented in Fig. 1(a), and the results are tabulated in table 1. Using Shannon-radii values for the ions [24] the value of the tolerance factor $t_{\mathrm{G}}$ for $\mathrm{HoCrO}_{3}$ is found to be $\sim 0.866$. Accordingly $\mathrm{HoCrO}_{3}$ is an orthorhombically distorted perovskite with the space group Pbnm [12 14]. The crystal structure at $300 \mathrm{~K}$ is in good agreement with the previously determined orthorhombic structure with similar lattice parameters [8,25]. The unit cell parameters obey the relationship, $a<c / \sqrt{2}<b$ which is characteristic of $O$-type orthorhombic structures. A buckling of the network of octahedra corresponding to cooperative rotation about a [110]-axis leads to the $O$-type orthorhombic structure. The clinographic view of the $\mathrm{CrO}_{6}$ octahedra in $\mathrm{HoCrO}_{3}$ is presented in Fig. 1(b). In perovskite manganites $R \mathrm{MnO}_{3}$ $(R=\mathrm{La}, \mathrm{Pr}, \mathrm{Nd}, \mathrm{Sm}, \mathrm{Eu}, \mathrm{Gd}, \mathrm{Tb}$ and $\mathrm{Dy})$, in addition to the distortion due to buckling of the $\mathrm{MnO}_{6}$ octahedron, a second distortion also arises because of the JahnTeller effect. This is because the $\mathrm{Mn}^{3+}$ in $R \mathrm{MnO}_{3}$ with four unpaired electrons the $d$-shell in high spin state is Jahn-Teller active. On the other hand $\mathrm{Cr}^{3+}$ in chromites with three unpaired electrons in $d$-orbitals is Jahn-Teller inactive. Thus a contribution to the lattice distortion in $\mathrm{RCrO}_{3}$ due to the Jahn-Teller effect is ruled out. 
Table 1: Atomic positions, unit cell dimensions and discrepancy factors of $\mathrm{HoCrO}_{3}$ obtained from the Rietveld refinement of the PXRD pattern at $300 \mathrm{~K}$. The values inside the brackets are the standard deviations.

\begin{tabular}{|c|c|c|c|c|}
\hline Atoms & $\mathrm{x}$ & $\mathrm{y}$ & $\mathrm{z}$ & $\mathrm{B}_{\text {iso }}\left(\AA^{2}\right)$ \\
\hline $\mathrm{Cr}$ & 0.5 & 0 & 0 & $0.815(2)$ \\
\hline Но & $-0.0168(8)$ & $0.0655(5)$ & 0.25 & $1.031(3)$ \\
\hline O1 & $0.1026(8)$ & $0.4664(8)$ & 0.25 & $1.037(5)$ \\
\hline $\mathrm{O} 2$ & $-0.3054(6)$ & $0.3055(6)$ & $0.0497(4)$ & $1.152(1)$ \\
\hline \multicolumn{5}{|c|}{ Unit Cell Dimensions } \\
\hline$a=5.2463(1) \AA$ & $b=5.5177(8) \AA$ & & 8) $\AA$ & $V=218.299(8) \AA^{3}$ \\
\hline \multicolumn{5}{|c|}{ Discrepancy Factors } \\
\hline$R_{p}=3.98 \%$ & $R_{w p}=5.53 \%$ & & $=3.06 \%$ & $\chi^{2}=3.27$ \\
\hline
\end{tabular}

\subsection{Specific heat}

The variation of the specific heat $\left(C_{\mathrm{P}}\right)$ of $\mathrm{HoCrO}_{3}$ with temperature is presented in Fig. 2. The low temperature features in $C_{\mathrm{P}}$ can be visualized clearly in a log-log plot as shown in the inset of Fig. 2. The direct inspection of the specific heat curve evidences the presence of three main contributions or features: (1) a sharp increase in specific heat below $2 \mathrm{~K}$ with a maximum at $\sim 0.3 \mathrm{~K}$ due to anomalously large hyperfine interaction between the electronic and nuclear spins of ${ }^{165} \mathrm{Ho}^{3+}$ leading to a nuclear-Schottky specific heat $\left(C_{\mathrm{N}}\right)$; $(2)$ the electronic Schottky contribution $\left(C_{e}\right)$ from thermal depopulation of the ${ }^{5} I_{8}$ ground state multiplet of $\mathrm{Ho}^{3+}$ with a maximum at $\sim 7 \mathrm{~K}$; and $(3)$ the $\lambda$-like anomaly with a peak at $T \approx 142 \mathrm{~K}$ due to the magnetic ordering of the $\mathrm{Cr}^{3+}$ moments. To determine different contributions to $C_{\mathrm{P}}$, a detailed analysis was performed in two steps, first $C_{\mathrm{P}}$ was modeled in the temperature range $0.1 \mathrm{~K} \leqslant T \leqslant 30 \mathrm{~K}$ and then $2 \mathrm{~K} \leqslant T \leqslant 290 \mathrm{~K}$.

\subsubsection{Specific heat in the temperature range $0.1 \mathrm{~K} \leqslant T \leqslant 30 \mathrm{~K}$}

The specific heat below $30 \mathrm{~K}$ has two broad features with maxima around $\sim 0.3 \mathrm{~K}$ and $\sim 7 \mathrm{~K}$. From the low temperature specific heat measurements of Ho metal it was found that anomalously large hyperfine interaction between the electronic and nuclear spins of Ho commonly leads to a nuclear Schottky anomaly with a maximum at $\approx 0.3 \mathrm{~K}[26$. The Hamiltonian for hyperfine interactions can be written in the form [27]:

$$
\frac{\mathscr{H}}{k_{\mathrm{B}}}=a^{\prime} I_{z}+P\left[I_{z}^{2}-\frac{1}{3} I(I+1)\right]
$$

where, $a^{\prime}$ is the magnetic hyperfine constant, which is a measure of the strength of the hyperfine interaction between the nuclear moment and the magnetic moment associated with the $4 f$ electrons. $P$ is the quadrupolar coupling constant. The field is applied in 
the $z$ direction. Since the projection $I_{z}$ can take $2 I+1$ values, i.e. $-I,-I+1, \ldots I$, the hyperfine specific heat $C_{\mathrm{P}}$ will be a Schottky type specific heat, associated with the $2 I+1$ hyperfine levels. The nuclear spin of ${ }^{165} \mathrm{Ho}$ with $100 \%$ natural abundance is $I=7 / 2$, while ${ }^{53} \mathrm{Cr}$ with $I=3 / 2$ has a natural abundance of $9.5 \%$. One can calculate the mean square of effective nuclear moment for the natural abundance of active isotopes of Ho and Cr, $\overline{\mu_{e f f}^{2}}$, yielding $22.4 \mu_{\mathrm{N}}^{2}$ for ${ }^{165} \mathrm{Ho}$ and $0.035 \mu_{\mathrm{N}}^{2}$ for Cr. With these effective nuclear moments one can conclude that the Cr hyperfine contribution is about three orders of magnitude smaller than the Ho one. Thus only the Ho contribution is taken into account in the calculation of nuclear hyperfine contribution to the specific heat. Due to the hyperfine interaction the holmium nucleus has $2 I+1=8$ possible spin orientations relative to an effective field $H_{\text {eff }}$. The energies $\varepsilon_{i} / k_{\mathrm{B}}$ of various nuclear spin states, i.e. the eigenvalues of the Hamiltonian in equation (11) are

$$
\frac{\varepsilon_{i}}{k_{\mathrm{B}}}=a^{\prime} i+P\left[i^{2}-\frac{1}{3} I(I+1)\right]
$$

where, $i=-7 / 2,-5 / 2, \ldots ., 5 / 2,7 / 2$. Information about $a^{\prime}$ and $P$ can be obtained by measuring the heat capacity at sufficiently low temperatures. In case of holmium, the quadrupolar coupling contribution is small and can be neglected [28]. Therefore for $P \approx 0$ the equation (2) reduces to,

$$
\frac{\varepsilon_{i}}{k_{\mathrm{B}}} \approx a^{\prime} i
$$

The specific heat in the temperature range $0.1-30 \mathrm{~K}$ is modeled by taking into consideration the contributions from nuclear specific heat $C_{\mathrm{N}}$, an electronic Schottky term $C_{e}$ and a lattice term $C_{\mathrm{L}}$. Thus at low temperatures the $C_{\mathrm{P}}$ of $\mathrm{HoCrO}_{3}$ is given by,

$$
C_{\mathrm{P}}=C_{\mathrm{N}}+C_{e}+C_{\mathrm{L}}
$$

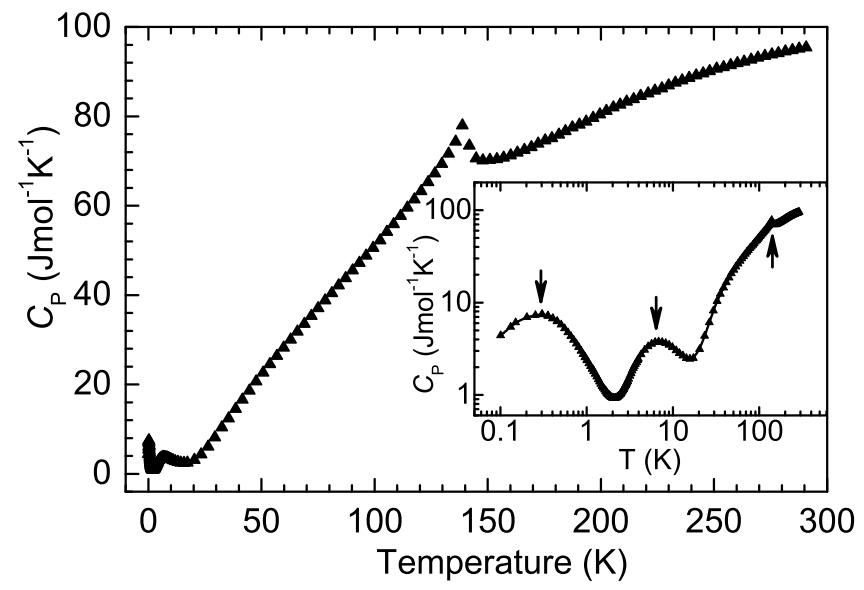

Figure 2: (Color online) The temperature dependence of specific heat $C_{\mathrm{P}}$ of $\mathrm{HoCrO}_{3}$. Three distinct features in the $C_{\mathrm{P}}$ are clearly visible in the inset. 

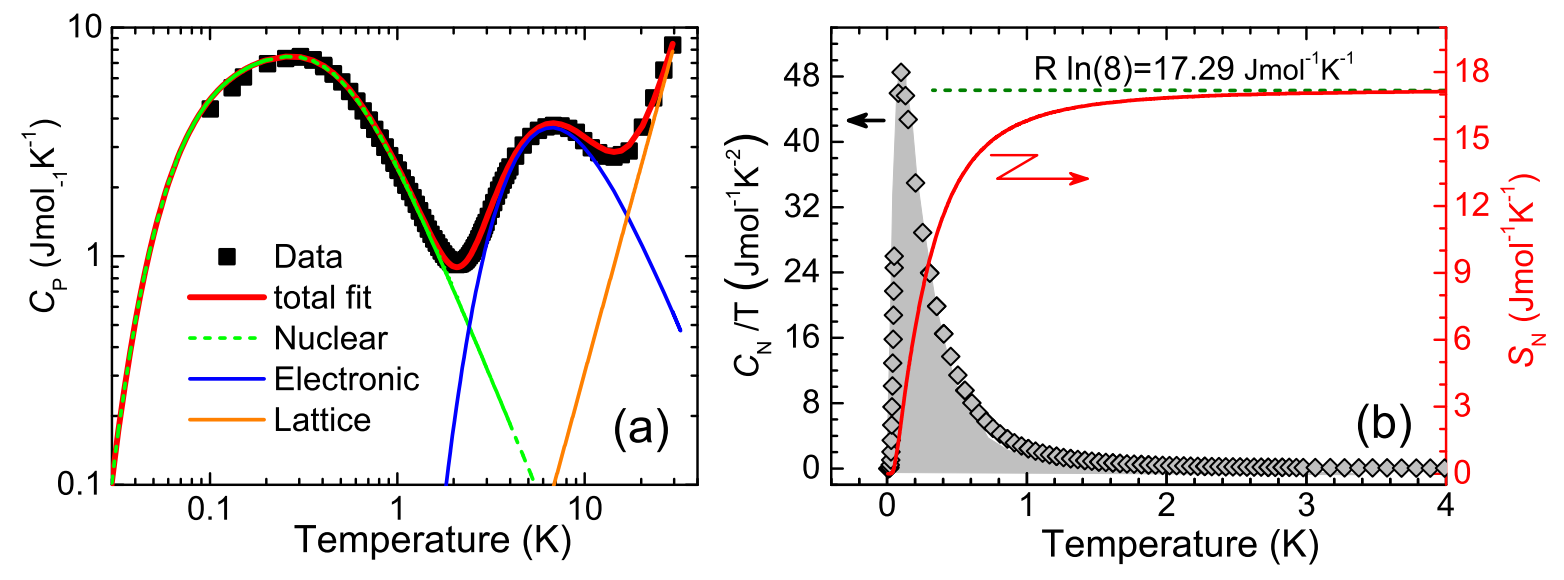

Figure 3: (Color online) (a) Double logarithmic plot of specific heat $C_{\mathrm{P}}$ measured at zero magnetic field plotted along with the refined model using equation 4. Different contributions to the total specific heat $C_{\mathrm{P}}$ are also shown. (b) A plot of $C_{\mathrm{N}} / T$ vs. T and the entropy associated with the nuclear specific heat $S_{\mathrm{N}}$ obtained by the numerical integration of $C_{\mathrm{N}} / T$ (shaded region) using equation 8. The horizontal dashed-line corresponds to the theoretical limiting value of the entropy.

where, $C_{\mathrm{N}}$ and $C_{e}$ are given by the general expression for an n-level Schottky specific heat term given by [29]:

$$
C_{\text {Schottky }}=\frac{R}{T^{2}} \frac{\sum_{i} \sum_{j}\left(\Delta_{i}^{2}-\Delta_{i} \Delta_{j}\right) \exp \left[-\left(\Delta_{i}+\Delta_{j}\right) / T\right]}{\sum_{i} \sum_{j} \exp \left[-\left(\Delta_{i}+\Delta_{j}\right) / T\right]}
$$

In this expression, $\Delta_{i}=\varepsilon_{i} / k_{\mathrm{B}}$ and $R=8.314 \mathrm{Jmol}^{-1} \mathrm{~K}^{-1}$ is universal gas constant. To calculate the nuclear specific heat $C_{\mathrm{N}}$, a Schottky curve for ${ }^{165}$ Ho with $I=7 / 2$, eight equally spaced energy levels with splitting energy, $\varepsilon_{i} / k_{\mathrm{B}} \approx a^{\prime} i$, where $i=$ $-7 / 2,-5 / 2, \ldots 5 / 2,7 / 2$, is used. To calculate the electronic Schottky specific heat $C_{e}$, a simple two level Schottky term with energy splitting $\varepsilon_{s} / k_{\mathrm{B}}$ is used, as the contribution from higher energy terms is negligible in the temperature range $0.1-30 \mathrm{~K}$. In this temperature range the lattice contribution is expressed by a single Debye term. At low temperatures, when $T<<\Theta_{\mathrm{D}}$, the Debye temperature, the Debye specific heat can be represented by well-known Debye $\mathrm{T}^{3}$-law as [29],

$$
C_{\text {Debye }}=R \frac{234 r_{\mathrm{D}} T^{3}}{\Theta_{\mathrm{D}}^{3}}=\beta_{3} T^{3}
$$

where, $r_{\mathrm{D}}$ is the number of atoms per molecule.

The fit results to equation (41) are given in Fig. 3 (a). From the fit the values of electronic Schottky splitting energy $\varepsilon_{s}$ and the Debye temperature $\Theta_{\mathrm{D}}$ are found to be, $1.379(5) \mathrm{meV}$ and $318(2) \mathrm{K}$, respectively. The Schottky energy $1.379(5) \mathrm{meV}$ is consistent with earlier reports based on specific heat measurements [16] and optical absorption Zeeman spectroscopy [30]. The value of $a^{\prime}$ from our analysis is found to 
be $0.2615(6) \mathrm{K}$ which is slightly smaller compared to the value found in metallic Ho, $a^{\prime} \approx 0.31-0.32 \mathrm{~K}\left[26,31,32\right.$, in inter-metallic $\mathrm{HoCo}_{2}, a^{\prime} \approx 0.31 \mathrm{~K}$ [33] and in paramagnetic salts, $a^{\prime} \approx 0.31 \mathrm{~K}[31$. The energy difference between two adjacent nuclear levels due to hyperfine field thus calculated from equation (3i) is $22.5(2) \mu \mathrm{eV}$. From inelastic neutron scattering measurements it is possible to observe these energy levels directly, as an inelastic peak centered at $\sim 22.5 \mu \mathrm{eV}$. Similar observations were made in spin-ice compound $\mathrm{Ho}_{2} \mathrm{Ti}_{2} \mathrm{O}_{7}$ which shows a nuclear Schottky peak around $0.3 \mathrm{~K}$ in the specific heat data [34], which was later observed in inelastic neutron scattering measurements as a peak at $\sim 26 \mu \mathrm{eV}$ [35]. The effective magnetic (hyperfine) field at the holmium nuclei can be computed by writing [32,

$$
a^{\prime}=\frac{\mu H_{e f f}}{k_{\mathrm{B}} I}
$$

where, $\mu=4.17 \mu_{\mathrm{N}}$ for ${ }^{165} \mathrm{Ho}$ and $\mu_{\mathrm{N}}$ is the nuclear magneton $\left(\mu_{\mathrm{N}}=5.051 \times 10^{-27} \mathrm{JT}^{-1}\right)$. Using the value of $a^{\prime}$ in equation (17) the hyperfine field, $H_{\text {eff }}$ is found to be 600(3) T. This value is comparable to the values reported for $\mathrm{Ho}_{2} \mathrm{Ti}_{2} \mathrm{O}_{7}(720 \mathrm{~T})$ [34, metallic Ho (770 T) [32] based on specific heat data and in $\mathrm{ErCrO}_{3}(530 \mathrm{~T})$ [36] based on Mössbauer spectroscopy.

The nuclear specific heat $C_{\mathrm{N}}$ was obtained by subtracting the lattice and electronic contributions from $C_{\mathrm{P}}$. The entropy $S_{\mathrm{N}}$ associated with the nuclear specific heat was calculated by the numerical integration of $C_{\mathrm{N}} / T . S_{\mathrm{N}}$ is given by the expression,

$$
S_{\mathrm{N}}(T)=\int_{0}^{T}\left(\frac{C_{\mathrm{N}}}{T}\right) d T
$$

The $C_{\mathrm{N}} / T$ versus $T$ and the computed $S_{\mathrm{N}}$ are presented in Fig. B(b). The nuclear entropy $S_{\mathrm{N}}$ reaches a maximum value of $\sim 17.2 \mathrm{Jmol}^{-1} \mathrm{~K}^{-1}$ at $\sim 5 \mathrm{~K}$. The theoretical limiting value of entropy for ${ }^{165} \mathrm{Ho}$ with nuclear spin $I=7 / 2$ is calculated as $\operatorname{Rln}(2 I+1)=$ $R \ln (8) \simeq 17.29 \mathrm{Jmol}^{-1} \mathrm{~K}^{-1}$. It is shown as an horizontal dashed line in Fig. 3(b). An excellent agreement between the experimental $S_{\mathrm{N}}$ with the theoretical value suggests that only contribution to the low temperature peak in the specific heat is from the nuclear Schottky term due to hyperfine interactions.

\subsubsection{Specific heat in the temperature range, $2 \mathrm{~K} \leqslant T \leqslant 290 \mathrm{~K}$.}

The non-magnetic contribution to the specific heat $C_{n m}$ in the temperature region $2-290 \mathrm{~K}$ is fitted assuming the contributions from an electronic Schottky term $C_{\text {Schottky }}$, lattice term $C_{\text {Lattice }}$ and a linear term $C_{\text {Linear }}$. $\mathrm{HoCrO}_{3}$ has 5 atoms per formula unit, which implies that 15 vibrational modes to the phononic specific heat exist. Taking in to account this constraint, we approximate the lattice contribution to the specific heat $\left(C_{\text {Lattice }}\right)$ as sum of a Debye term $\left(C_{\text {Debye }}\right)$ and two distinct Einstein terms $C_{\text {Einstein }}$. The specific heat associated with the magnetic ordering, $\left(C_{\mathrm{m}}\right)$ results in a $\lambda$-like transition with a maximum at $\sim 142 \mathrm{~K} . C_{\mathrm{m}}$ is obtained by subtracting $C_{n m}$ from the experimental data $\left(C_{\mathrm{P}}\right)$. The magnetic entropy $S_{\mathrm{m}}$ associated with $C_{\mathrm{m}}$ is obtained by the numerical 
integration of $C_{\mathrm{m}} / T$. The $C_{n m}$ and $C_{\mathrm{m}}$ in the temperature range $2-290 \mathrm{~K}$ can be written as [29, 37],

$$
\begin{aligned}
& C_{n m}=C_{\text {Schottky }}+C_{\text {Lattice }}+C_{\text {Linear }} \\
& C_{\text {Lattice }}=C_{\text {Debye }}+C_{\text {Einstein }} \\
& C_{\mathrm{m}}=C_{\mathrm{P}}-C_{n m}
\end{aligned}
$$

Here $C_{\text {Schottky }}$ is purely electronic Schottky term as we are fitting only above $2 \mathrm{~K}$, where the nuclear contribution is negligible. The non-magnetic contribution to the specific heat in this temperature is obtained by equations (5), (9), (10) and,

$$
\begin{aligned}
& C_{\text {Debye }}=9 r R / x_{\mathrm{D}}^{3} \int_{0}^{x_{\mathrm{D}}} x^{4} e^{x} /\left(e^{x}-1\right)^{2} d x \\
& C_{\text {Einstein }}=3 r R \sum_{i} a_{i}\left[x_{i}^{2} e^{x_{i}} /\left(e^{x_{i}}-1\right)^{2}\right] \\
& C_{\text {Linear }}=\gamma T
\end{aligned}
$$

In these expressions, $R$ is the gas constant, $x_{\mathrm{D}}=\hbar \omega_{\mathrm{D}} / k_{\mathrm{B}} T, x_{i}=\hbar \omega_{\mathrm{E}} / k_{\mathrm{B}} T, k_{\mathrm{B}}$ is Boltzmann constant, $\gamma$ is the coefficient of the linear term and $r$ is the number of atoms per molecule. The fitting was performed excluding the data in the temperature range $60-200 \mathrm{~K}$. The different contributions to the measured specific heat obtained from fitting are presented in Fig. 4(a). The values of Debye temperature $\Theta_{\mathrm{D}}$ and two Einstein temperatures $\left(\Theta_{E 1}, \Theta_{E 2}\right)$ obtained from the fit are 538(14) $\mathrm{K}(46(1) \mathrm{meV})$ and $784(15) \mathrm{K}$ $\left(67(1) \mathrm{meV}\right.$ ) and $176(2) \mathrm{K}(15.17(2) \mathrm{meV})$, respectively. This contribution to $C_{\text {Lattice }}$ was parametrized by using 3 Debye modes with Debye temperature $\left(\Theta_{\mathrm{D}}\right), 7$ Einstein modes with Einstein temperature $\Theta_{E 1}$, and another 5 Einstein modes with $\Theta_{E 2}$. Although the used parametrization over simplify the phonon spectrum, the obtained key results are not influenced by subtleties in the choice of the modeled lattice contribution, i.e., by the number of Debye and Einstein contribution or by the used absolute values within reasonable error bars [38].

Based on optical absorption spectroscopy [30, magnetization and magnetic susceptibility [17], specific heat [39], elastic neutron diffraction [40] and inelastic neutron scattering (INS) [1] experiments five electronic Schottky levels were observed in $\mathrm{HoCrO}_{3}$ with the fifth level being at $\sim 272 \mathrm{~K}(23.4 \mathrm{meV})$. To calculate $C_{\text {Schottky }}$, a Schottky curve for five energy levels is used which contribute to $C_{\text {Schottky }}$ below $300 \mathrm{~K}$. The ground state energy level is assumed to be zero i.e., $\varepsilon_{1} / k_{\mathrm{B}}=0$. From the low temperature specific heat analysis, where a simple two level Schottky model was used to calculate $C_{S c h o t t k y}$, the energy splitting is found to be $1.379(5) \mathrm{meV}$. We fixed this value as $\varepsilon_{2}$ in the present calculations. The higher energy levels are determined from the fit as $\varepsilon_{3}=10.37(4) \mathrm{meV}$, $\varepsilon_{4}=15.49(9) \mathrm{meV}$ and $\varepsilon_{5}=23.44(9) \mathrm{meV}$. These values are in excellent agreement with the reported crystal field energy values in $\mathrm{HoCrO}_{3}$ (within 0.5\%).

After subtracting the contributions, $C_{\text {Schottky }}, C_{\text {Lattice }}$ and $C_{\text {Linear }}$ from the total specific heat $C_{\mathrm{P}}$, the magnetic specific heat $C_{\mathrm{m}}$ is obtained, which can be seen as the deviation from the total fit in Fig. 4(a) in the temperature range $60-180 \mathrm{~K}$. The magnetic entropy, $S_{\mathrm{m}}$ was calculated by the numerical integration of $C_{\mathrm{m}} / T$ analogous 

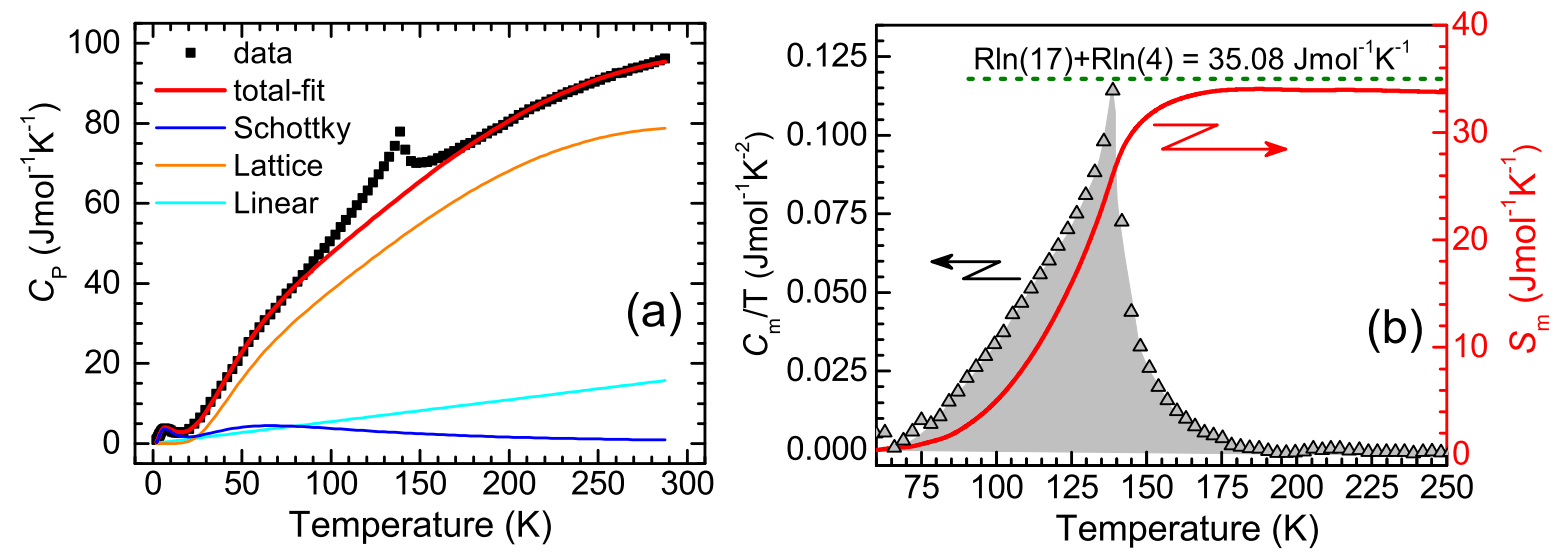

Figure 4: (Color online) (a) Specific heat, $C_{\mathrm{P}}$ measured at zero magnetic field plotted along with the fitted results using the equation (9). Different contributions to the total specific heat $C_{\mathrm{P}}$ are also shown. (b) The $C_{\mathrm{m}} / \mathrm{T}$ vs. T and the magnetic entropy $S_{\mathrm{m}}$, obtained by the numerical integration of $C_{\mathrm{m}} / T$ (shaded region). The horizontal dashedline corresponds to the theoretical limiting value of the entropy.

to the expression (8), by replacing $C_{\mathrm{N}}$ by $C_{\mathrm{m}}$. The $C_{\mathrm{m}} / T$ versus $T$ and computed $S_{\mathrm{m}}$ is presented in Fig. 4(b). The experimental magnetic entropy value reaches a maximum value of $\sim 34 \mathrm{Jmol}^{-1} \mathrm{~K}^{-1}$ around $180 \mathrm{~K}$, above $T_{\mathrm{N}}$. The theoretical limiting value of $S_{\mathrm{m}}$ was calculated by adding the contributions due to ordering of both $\mathrm{Ho}^{3+}(J=8)$ and $\mathrm{Cr}^{3+}(S=3 / 2)$ moments, i.e., $R \ln (17)+R \ln (4) \simeq 35.08 \mathrm{Jmol}^{-1} \mathrm{~K}^{-1}$, indicated as a horizontal dotted line in Fig. 4(b). The experimental magnetic entropy is very close to the theoretical magnetic entropy around $180 \mathrm{~K}(\sim 97 \%)$. The good agreement between the experimentally found value for the magnetic entropy and that calculated for the spin only component of $\mathrm{Cr}^{3+}$ ions and orbital magnetic moment of $\mathrm{Ho}^{3+}$ ions, allows for concluding the following: first, the orbital moments of $\mathrm{Cr}^{3+}$ ions appear to be quenched while keeping the full spin moment. Second, the lattice contribution seems to be described sufficiently well by the Debye and Einstein models.

The value of linear coefficient $\gamma$ from the fit is found to be $6.3(8) \mathrm{mJmol}^{-1} \mathrm{~K}^{-2}$. This value of $\gamma$ is comparable to the reported values, $5-7 \mathrm{mJmol}^{-1} \mathrm{~K}^{-2}$ which is associated with conduction electrons in some doped manganites [42 45. The linear coefficient is usually attributed to charge carriers, and is proportional to the density of states at the Fermi level. However, $\mathrm{HoCrO}_{3}$ is an electrical insulator, thus the origin of linear term should be interpreted with caution. Several electrical insulators have been reported with large values of $\gamma$, viz., $\mathrm{LaMnO}_{3+\delta}\left(\gamma \approx 20 \mathrm{mJmol}^{-1} \mathrm{~K}^{-2}\right)$ [46], $\mathrm{BaVS}_{3}$ $\left(\gamma=15.7 \mathrm{mJmol}^{-1} \mathrm{~K}^{-2}\right)$ [47] and $\mathrm{La}_{2.3} \mathrm{YCa}_{0.7} \mathrm{Mn}_{2} \mathrm{O}_{7}\left(\gamma=41.5 \mathrm{mJmol}^{-1} \mathrm{~K}^{-2}\right)$ [48]. The origin of a linear contribution in these electrical insulators was attributed to a variety of magnetic phenomena. The most plausible explanation for the appearance of a linear term in the heat capacity in $\mathrm{HoCrO}_{3}$ is due to disordered $\mathrm{Ho}^{3+}$ as in the case of insulating $\mathrm{Ho}_{1-x} \mathrm{Y}_{x} \mathrm{MnO}_{3}$. For this compound it was observed that with increasing $\mathrm{Y}$ content $\gamma$ is reduced and drops to zero at $x=0.9$, indicating the dependence of $\gamma$ on 
the Ho content [49]. It was interpreted that the appearance of the linear term is due to high degeneracy of disordered $\mathrm{Ho}^{3+}$ spins above the ordering temperature. From high resolution neutron spectroscopy a huge quasielastic scattering was observed in $\mathrm{HoCrO}_{3}$, which was understood as due to fluctuating disordered Ho electronic moments [22]. This supports our interpretation of disordered Ho electronic moments as a possible source of a linear term in the observed specific heat. It is worth noting at this point that, to model the low temperature specific heat data using equation (44), a linear term was not required which can be understood due to spin ordering of $\mathrm{Ho}^{3+}$ at these low temperatures [50. These observations confirm that the main origin of the linear term in $\mathrm{HoCrO}_{3}$ is disordered $\mathrm{Ho}^{3+}$ spins.

\subsection{Inelastic neutron scattering}

Inelastic neutron scattering spectra measured at the BASIS back-scattering spectrometer are presented in Fig. 5 (a). At low temperatures two clear inelastic signals are observed on both energy gain and energy loss sides. A detailed study of the hyperfine spectra of $\mathrm{HoCrO}_{3}$ is published elsewhere [22]. The inelastic spectra was modeled with the equation,

$$
S(\omega)=\left[x \delta_{e l}(\omega)+p_{1} \delta_{i n s 1}\left(-\omega_{1}\right)+p_{2} \delta_{i n s 2}\left(+\omega_{2}\right)\right] \otimes R(\omega)+B
$$

where, delta function $\delta_{e l}$ and $\delta_{i n s}$ represent elastic and inelastic peaks, respectively. These terms are convoluted numerically with the experimentally determined resolution function, $R(\omega)$, which is asymmetric due to the neutron pulse shape. $B$ is a flat background term and $x, p_{1}$ and $p_{2}$ are scaling factors. The average energies of the inelastic peaks as obtained from the fits are $E= \pm 22.18(4) \mu \mathrm{eV}$, this is in excellent agreement with the hyperfine splitting energy, 22.5(2) $\mu \mathrm{eV}$ determined from our low temperature specific heat data. The fitting result to the $1.5 \mathrm{~K}$ data is presented in Fig. 馬(b). As can be seen from Fig. (5)(a) a strong quasielastic scattering signal arises with increasing temperatures, broadening the elastic peak. An additional Lorentzian term was required to describe this intensity. The temperature evolution of the quasielastic term was attributed to fluctuating electronic moments of the Ho ions, which get increasingly disordered at higher temperatures. This reassures the validity of the large linear coefficient obtained by fitting the specific heat data at higher temperatures. From our earlier detailed report on the temperature dependence quasielastic scattering in $\mathrm{HoCrO}_{3}$ 222] we found that the intensity decreases sharply below $40 \mathrm{~K}$. Further the temperature dependence of ordered magnetic moment of Ho obtained from our recent neutron powder diffraction measurements [50], varies inversely as the temperature dependence of quasielastic scattering intensity and shows a sharp increase below $40 \mathrm{~K}$. This confirms that the origin of quasielastic scattering is indeed fluctuating Ho moments which are short range in nature. A similar phenomenon was also observed in $\mathrm{Ho}_{2} \mathrm{Ti}_{2} \mathrm{O}_{7}$, the authors have interpreted it as being due to the fluctuating electronic moments of the Ho [35]. Wan et al., [51] showed both analytically and numerically that indirect magnetic exchange, which is short-range in nature is another driving force for the off-center atomic 

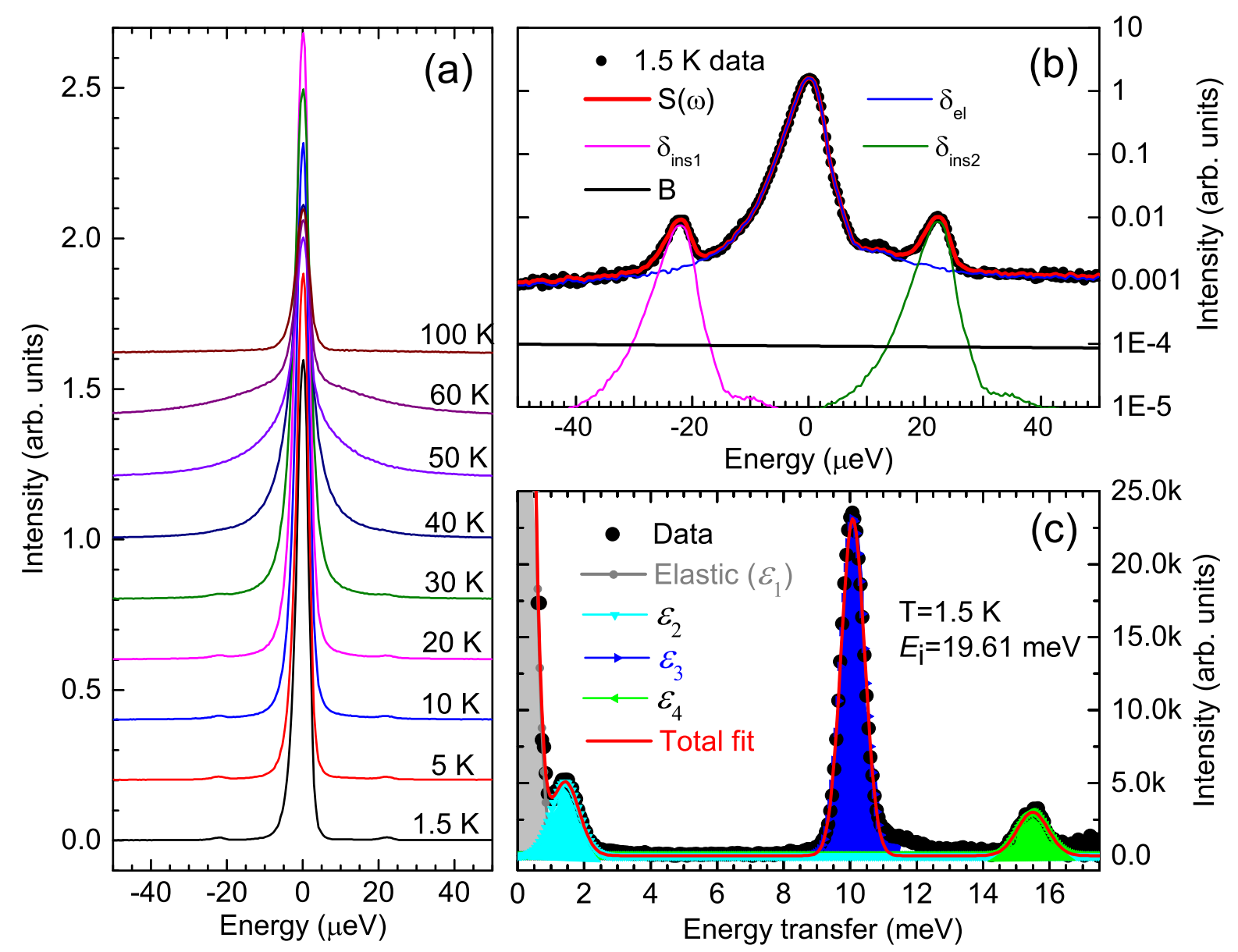

Figure 5: (Color online) (a) INS spectra of $\mathrm{HoCrO}_{3}$ collected on the instrument BASIS, spectral lines are shifted along y-axis for clarity. (b) Fits to the $1.5 \mathrm{~K}$ INS data as described in the main text using equation (15). The inelastic peaks at both energy loss and energy gain sides are fitted by convoluting the instrument resolution function determined from vanadium with two delta functions for two inelastic peaks (magenta and green lines) plus one delta function for the elastic peak (blue line). The horizontal line is the flat background term [22]. (c) INS spectra collected on the instrument FOCUS, with neutrons of incident energy $E_{i}=19.61 \mathrm{meV}$ at temperature $1.5 \mathrm{~K}$. Three well-resolved peaks at $\varepsilon_{2}, \varepsilon_{3}$ and $\varepsilon_{4}$ are fitted using a Gaussian peak function.

motion and ferroelectricity. In the present case, proposed short-range magnetic exchange interactions could cause the off-center atomic motion leading to ferroelectricity. We will report a detailed nuclear and magnetic structure studies as a function of temperature elsewhere, which should shed more light on atomic displacements and short range magnetic order in $\mathrm{HoCrO}_{3}$.

A typical inelastic spectrum measured at the time-of-flight instrument FOCUS is presented in Fig. 5)(c). The non-Kramer's $\mathrm{Ho}^{3+}$ ions in $\mathrm{HoCrO}_{3}$ are at sites of point group symmetry $m\left(\mathrm{C}_{1 \mathrm{~h}}\right)$, which typically leads to a singlet ground state. Thus the ground multiplet of the $\mathrm{Ho}^{3+}$ ion, ${ }^{5} I_{8}$, split into $2 J+1=17$ singlets by the crystalline 

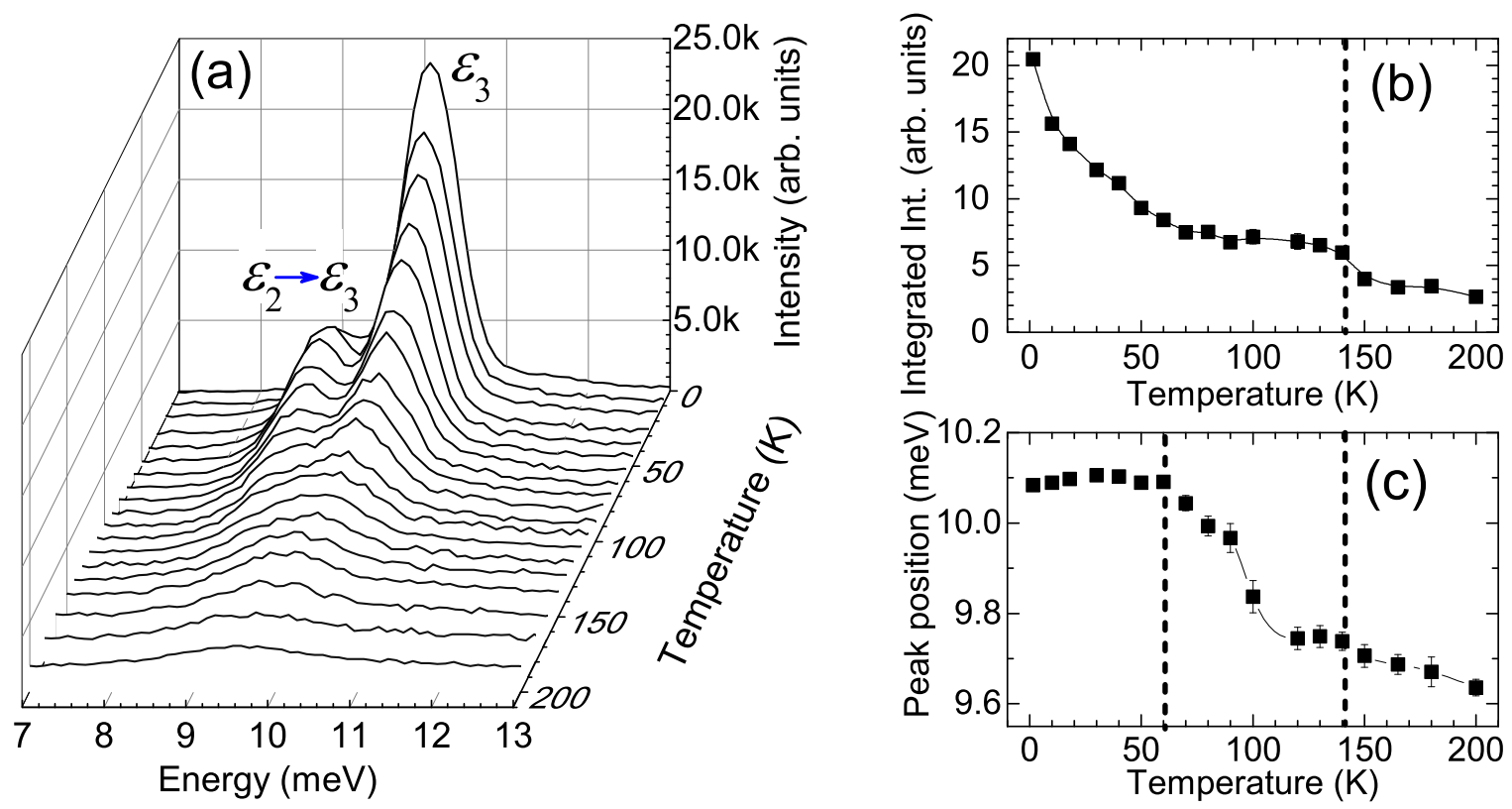

Figure 6: (a) Temperature dependence of Crystalline electric field excitations with the energy range 7-13 meV. (b) Temperature dependence of integrated intensity of peak corresponding to CEF excitation $\varepsilon_{3}$ and (c) Temperature dependence of peak position, for the inelastic peak corresponding to crystal electric field excitation $\varepsilon_{3}$. Vertical dashed line at $140 \mathrm{~K}$ indicates the magnetic ordering temperature and the dashed line at $60 \mathrm{~K}$ corresponds to the peak in pyroelectric current curve, reported in reference [8].

field generated by surrounding ions. The energy range of our inelastic data, limited to only three crystal field levels, makes a crystal field calculation inadequate using a point-charge model. Despite that, the observed inelastic peaks, fitted with Gaussian peak functions as shown in Fig. 5)(c), are centered at energies, $\varepsilon_{2}=1.45(6) \mathrm{meV}$, $\varepsilon_{3}=10.07(2) \mathrm{meV}$ and $\varepsilon_{4}=15.49(2) \mathrm{meV}$ are in excellent agreement with those determined from heat capacity data and reported values [41.

The hyperfine excitation signal which is masked gradually by quasielastic scattering upon increasing temperature, in contrast, the Crystal Electric Field (CEF) excitation signal is still visible in high temperature range. The temperature evolution of inelastic peak associated with the CEF excitation between ground state $\left(\varepsilon_{1}\right)$ and the second excitation level $\left(\varepsilon_{3}\right)$ is presented in Fig. 6(a). The inelastic peak due to transitions between first excitation level $\left(\varepsilon_{2}\right)$ and the second excitation level $\left(\varepsilon_{3}\right)$ can also be seen (above $10 \mathrm{~K}$ ). Two inelastic peaks were simultaneously fitted with Gaussian peak function, both the peaks are well separated in all temperatures below $100 \mathrm{~K}$ above which the peak due to the transition between first excitation level $\left(\varepsilon_{2}\right)$ and the second excitation level $\left(\varepsilon_{3}\right)$ vanishes. The integrated peak intensity as a function of temperature for the peak corresponding to crystal field excitation $\varepsilon_{3}$ is plotted in Fig. 6(b). It exhibits a typical behavior of Van Vleck contribution below $T_{\mathrm{N}}$ and a clear anomaly at $T_{\mathrm{N}}=140 \mathrm{~K}$. Given the fact that the CEF interaction reflects directly the electrical and 
magnetic potential created by neighboring ions, the anomaly of peak intensity around $T_{\mathrm{N}}$ indicated the change of local environment surrounding the $\mathrm{Ho}^{3+}$ ion in $\mathrm{HoCrO}_{3}$. Because both $\mathrm{Cr}$ and Ho moments order below $T_{\mathrm{N}}$, the anomaly on peak intensity can be mainly attributed to the effect of exchange field on the $\mathrm{Ho}^{3+}$ ion from the long range order of $\mathrm{Cr}^{3+}$ magnetic sublattice. Rajeswaran et al., [5 proposed that the multiferroicity in $R \mathrm{CrO}_{3}$ is caused by the interaction between magnetic rare earth and weak ferromagnetic $\mathrm{Cr}^{3+}$ ions following the breaking of symmetry. The observed anomaly in CEF signal strengthens the importance of Ho-Cr exchange striction. The anomaly in 6(b) also implies the possible distortion of Ho ions and their surroundings since the pyroelectric current exhibits its maximal at the same temperature [8].

The temperature dependence of $\varepsilon_{3}$ peak position is shown in Fig. 6(c). In the temperature range $1.5-60 \mathrm{~K}$, the peak position remains unchanged at $10.1 \mathrm{meV}$ and starts moving gradually to lower energy, above $\sim 60 \mathrm{~K}$, which is well below $T_{\mathrm{N}}$. The change of peak position of CEF excitation at $\sim 60 \mathrm{~K}$ hints at the shift of CEF levels accompanied with the change of local crystallographic symmetry of $\mathrm{Ho}^{3+}$ ion. However, so far there is no report on the observation of structural distortion for $\mathrm{HoCrO}_{3}$ at $60 \mathrm{~K}$. It is noticed that a tiny peak is observed at the same temperature in pyroelectric current curve, as shown in Fig. 5(a) in the ref. [8]. Therefore, the change of CEF peak position at $60 \mathrm{~K}$ is related to the change of ferroelectric properties. A detailed study on the temperature-dependent crystal structure is needed to understand the unusual behavior of CEF excitation and thus the mechanism of multiferroicity in $\mathrm{HoCrO}_{3}$.

\section{Summary and conclusions}

High quality polycrystalline $\mathrm{HoCrO}_{3}$ was prepared by solid state reaction method and characterized by means of x-ray powder diffraction, heat capacity and inelastic neutron scattering measurements. From the structural analysis we could establish the consistency of the observed crystal structure and theoretical predictions based on Goldschimdt's tolerance factor rule. From the low temperature nuclear contribution to the specific heat results we obtained the first CEF excitation energy for $\mathrm{Ho}^{3+}$, $1.379(5) \mathrm{meV}$ and hyperfine field of $600(3) \mathrm{T}$ with a hyperfine splitting energy, $22.5(2) \mu \mathrm{eV}$ for ${ }^{165} \mathrm{Ho}$ with $I=7 / 2$. The entropy $\left(S_{\mathrm{N}}\right)$ associated with nuclear hyperfine specific heat $\left(C_{\mathrm{N}}\right)$ was also estimated from the low temperature specific heat, which is in excellent agreement with theory. The hyperfine splitting energy determined from specific heat data is then confirmed from the peak observed in inelastic back scattering data. The large linear term $\gamma=6.3(8) \mathrm{mJmol}^{-1} \mathrm{~K}^{-2}$ in the specific heat was understood as due to disordered $\mathrm{Ho}^{3+}$ spins, which is further supported by strong quasielastic scattering observed in inelastic backscattering data at high temperatures. From the analysis of high temperature specific heat, by fixing the first CEF excitation level to $1.379(5) \mathrm{meV}$, obtained from low temperature specific heat analysis, we determined three more crystal field transitions at $10.37(4) \mathrm{meV}, 15.49(9) \mathrm{meV}$ and $23.44(8) \mathrm{meV}$. The lower energy crystal field levels observed from the inelastic neutron scattering 
measurements are in excellent agreement with those determined from specific heat data. The magnetic entropy $\left(S_{\mathrm{m}}\right)$ associated with the magnetic specific heat $\left(C_{\mathrm{m}}\right)$ is obtained, which is consistent with the theoretical prediction. The linear term in specific heat and also quasielastic scattering observed in inelastic neutron spectra, further adds another possible driving force for the observed ferroelectricity in the form of short range exchange interactions in this compound as proposed by Wan et al., [51]. Further, from the temperature evolution of crystal field spectra we confirm a direct correlation between the magnetic ordering and the ferroelectricity in this compound, as predicted by Rajeswaran et al. [5]. In addition to aforementioned mechanisms, the asymmetry driven ferroelectricity as proposed by Ghosh et al. [8, 10, and Indra et al., [11] should also be considered as one of the driving force for ferroelectricity. More detailed temperature dependent structural studies are required for the quantitative analysis of distortions induced ferroelectricity. Thus our study and recent reports on the ferroelectricity confirm that $\mathrm{HoCrO}_{3}$ and $\mathrm{RCrO}_{3}$ in general, possesses more than one ingredient which can drive ferroelectricity, suggesting that these materials are potential multiferroic candidates for device applications. Our study warrants, more detailed temperature dependent nuclear and magnetic structure studies to establish a most favorable mechanism for multiferroicity in $\mathrm{HoCrO}_{3}$ and rare-earth orthochromites in general.

\section{Acknowledgments}

We thank the expert assistance of T. Strässle, SINQ, Paul Scherrer Institute. Part of the research conducted at SNS was sponsored by the Scientific User Facilities Division, Office of Basic Energy Sciences, US Department of Energy. This work is partially based on the experiments performed at the Swiss Spallation Neutron Source SINQ, instrument FOCUS (Proposal ID 20090536). Financial support from the European Project EU NMI3 is acknowledged.

\section{References}

[1] Serrao C R, Kundu A K, Krupanidhi S B, Waghmare U V and Rao C N R 2005 Phys. Rev. B 72 220101

[2] Sahu J R, Serrao C R, Ray N, Waghmare U V and Rao C N R 2007 J. Mater. Chem. 1742

[3] Su Y, Zhang J, Feng Z, Li Z, Shen Y and Cao S 2011 J. Rare Earths 291060

[4] Benedek N A, Mulder A T and Fennie C J 2012 J. Solid State Chem. 19511

[5] Rajeswaran B, Khomskii D I, Zvezdin A K, Rao C N R and Sundaresan A 2012 Phys. Rev. B 86(21) 214409

[6] Preethi Meher K R S, Wahl A, Maignan A, Martin C and Lebedev O I 2014 Phys. Rev. B 89(14) 144401

[7] Apostolov A T, Apostolova I N and Wesselinowa J M 2015 Mod. Phys. Lett. B 291550251

[8] Ghosh A, Pal A, Dey K, Majumdar S and Giri S 2015 J. Mater. Chem. C 3(16) 4162

[9] Subba Rao G V, Chandrashekhar G V and Rao C N R 1968 Solid State Commun. 6177

[10] Ghosh A, Dey K, Chakraborty M, Majumdar S and Giri S 2014 EPL 10747012 
[11] Indra A, Dey K, Midya A, Mandal P, Gutowski O, Rütt U, Majumdar S and Giri S 2016 J. Phys.: Condens. Matter 28166005

[12] Geller S and Wood E A 1956 Acta Crystallogr. 9563

[13] Geller S 1956 J. Chem. Phys. 241236

[14] Bertaut E F and Forrat F 1956 J. Phys. (Paris) 17129

[15] Bertaut E, Mareschal J, De Vries G, Aleonard R, Pauthenet R, Rebouillat J and Zarubicka V 1966 IEEE Trans. Magn. 2453

[16] Pataud P and Sivardière J 1970 J. de Phys. 31803

[17] Hornreich R M, Wanklyn B M and Yaeger I 1972 Int. J. Magn. 277

[18] Rietveld H M 1969 J. Appl. Crystallogr. 265

[19] Rodríguez-Carvajal J 1993 Physica B 19255

[20] Hwang J S, Lin K J and Tien C 1997 Rev. Sci. Instrum. 6894

[21] Mamontov E and Herwig K W 2011 Rev. Sci. Instrum. 82085109

[22] Chatterji T, Jalarvo N, Kumar C M N, Xiao Y and Brückel T 2013 J. Phys. Condens. Matter 25 286003

[23] Azuah R, Kneller L, Qiu Y, Tregenna-Piggott P, Brown C, Copley J and Dimeo R 2009 J. Res. Natl. Inst. Stand. Technol. 114341

[24] Shannon R D 1976 Acta Crystallogr., Sect. A: Found. Crystallogr. 32751

[25] Quezel-Ambrunaz S and Mareschal J 1963 Bull. Soc. Fr. Mineral. Crystallogr. 36204

[26] Van Kempen H, Miedema A R and Huiskamp W J 1964 Physica 30229

[27] Bleaney B and Hill R W 1961 Proc. Phys. Soc. London 78313

[28] Rossat-Mignod J, Quezel G, Berton A and Chaussy J 1974 Phys. Status Solidi B 63105

[29] Tari A 2003 The specific heat of matter at low temperatures (Imperial College Press, London)

[30] Courths R and Hüfner S 1976 Zeitschrift für Physik B Condensed Matter 24193

[31] Gordon J E, Dempesy C W and Soller T 1961 Phys. Rev. 124724

[32] Lounasmaa O V 1962 Phys. Rev. 1281136

[33] Bloch D, Voiron A, Berton A and Chaussy J 1973 Solid State Commun. 12685

[34] Jana Y M and Ghosh D 2000 Phys. Rev. B 619657

[35] Ehlers G, Mamontov E, Zamponi M, Kam K C and Gardner J S 2009 Phys. Rev. B 102016405

[36] Eibschütz M, Cohen R L and West K W 1969 Phys. Rev178 572

[37] Gopal E S R 1966 Specific heats at low temperatures (Heywood books London)

[38] Schäpers M, Wolter A U B, Drechsler S L, Nishimoto S, Müller K H, Abdel-Hafiez M, Schottenhamel W, Büchner B, Richter J, Ouladdiaf B, Uhlarz M, Beyer R, Skourski Y, Wosnitza J, Rule K C, Ryll H, Klemke B, Kiefer K, Reehuis M, Willenberg B and Süllow S 2013 Phys. Rev. B 88(18) 184410

[39] Pataud P and Sivardière J 1970 J. de Phys. 311017

[40] Shamir N, Shaked H and Shtrikman S 1977 Physica B+C 90211

[41] Shamir N, Melamud H, Shaked H and Shtrikman S 1977 Physica B 90217

[42] Ghivelder L, Abrego Castillo I, Alford N, Tomka G J, Riedi P, MacManus-Driscoll J, Akther Hossain A and Cohen L 1998 J. Magn. Magn. Mater. 189274

[43] Hamilton J J, Keatley E L, Ju H L, Raychaudhuri A K, Smolyaninova V N and Greene R L 1996 Phys. Rev. B 54(21) 14926

[44] Coey J M D, Viret M, Ranno L and Ounadjela K 1995 Phys. Rev. Lett. 75(21) 3910

[45] Woodfield B F, Wilson M L and Byers J M 1997 Phys. Rev. Lett. 78(16) 3201

[46] Ghivelder L, Abrego Castillo I, Gusmao M A, Alonso J A and Cohen L F 1999 Phys. Rev. B 60 12184

[47] Imai H, Wada H and Shiga M 1996 J. Phys. Soc. Jpn. 653460

[48] Raychaudhuri P, Mitra C, Paramekanti A, Pinto R, Nigam A K and Dhar S K 1998 J. Phys. Condens. Matter 10 L191

[49] Zhou H D, Lu J, Vasic R, Vogt B W, Janik J A, Brooks J S and Wiebe C R 2007 Phys. Rev. B 75132406 
[50] Kumar C M N, Xiao Y, Nandi S, Senyshyn A, Su Y, Brückel T Magnetic ordering and magnetoelastic effect in $\mathrm{HoCrO}_{3}$, manuscript in preparation

[51] Wan X, Ding H C, Savrasov S Y and Duan C G 2016 Scientific reports 622743 